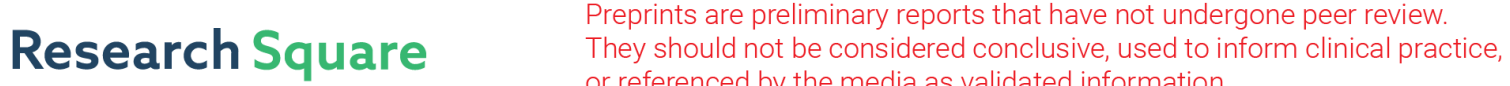 or referenced by the media as validated information. \\ Nonlinear Mixing of Two Laser Beams and THz Plasmons Generation in Graphene Coated Optical Fibre
}

Neha Verma

MMH College, Ghaziabad

Anil Govindan

MMH College, Ghaziabad

Pawan Kumar ( $\square$ kumarpawan_30@yahoo.co.in )

IIT Delhi https://orcid.org/0000-0001-5710-0203

\section{Research Article}

Keywords: Nonlinear, Laser Beams, THz plasmons, optical Fibre, space communications, biomedical imaging, tomography, precision radar

Posted Date: January 3rd, 2022

DOI: https://doi.org/10.21203/rs.3.rs-1079347/v1

License: (c) (i) This work is licensed under a Creative Commons Attribution 4.0 International License. Read Full License 


\title{
Nonlinear Mixing of Two Laser Beams and THz plasmons generation in graphene coated optical Fibre
}

\author{
Neha Verma, Anil Govindan \\ Department of Physics, MMH College Ghaziabad, UP, India \\ Pawan Kumar* \\ Department of physics, Raj Kumar Goel Institute of Technology, Ghaziabad, UP- 201003, India \\ Research Centre for Compact Radiation Sources, Raj Kumar Goel Institute of Technology \\ Ghaziabad, UP- 201003, India

\begin{abstract}
ABSTARCT
Graphene is a most suitable material for Terahertz $(\mathrm{THz})$ radiation generation. An efficient mechanism of $\mathrm{THz}$ surface plasmons (THz SPs) generation in graphene coated optical fibre by nonlinear mixing of two laser beams is proposed. The graphene coated fiber supports THz SPs with plasmon resonance in the $\mathrm{THz}$ regime and controllable by thickness of graphene and radius of optical fibre. The laser beams exert a difference frequency ponderomotive force on the electrons of the graphene. This ponderomotive force induces a nonlinear current in graphene which driving the difference frequency $\mathrm{THz}$ SPs. The normalized amplitude of $\mathrm{THz}$ SPs decreases with frequency as the nonlinear coupling gets weaker. The efficiency of the device is around $0.01 \%$ at a laser intensity of $3 \times 10^{14} \mathrm{~W} / \mathrm{cm}^{2}$. This scheme will be useful making the compact $\mathrm{THz}$ radiation source and $\mathrm{THz}$ plasmon sensor.

*Corresponding Author: kumarpawan_30@yahoo.co.in
\end{abstract}




\section{Introduction}

There is a substantial interest of terahertz radiation generation due to its wide applications in outer space communications, biomedical imaging, tomography, precision radar and other areas [1-7]. Due to the lack of efficient sources and detectors in THz range or so called $\mathrm{THz}$ gap (0.1$10 \mathrm{THz}$ ), however, this $\mathrm{THz}$ gap is still remains a challenge for modern technology and poses significant limitations for further developments in this field. These limitations, arising due to the nature of light/material interaction at terahertz frequencies, are imprinted also onto the design of terahertz systems for light control, manipulation, and, most significantly, radiation guidance.

The laser based $\mathrm{THz}$ radiation sources are attractive due to relative simplicity and suitability for modest $\mathrm{THz}$ power [8-9]. However since for most applications, size is major constraints a number of techniques that exploit short pulse laser- semiconductor interaction to produce $\mathrm{THz}$ radiation by transient photoconductors or dc current modulation have developed [10-11]. Intense short pulse laser plasma interaction also offers potential schemes [12-15] for the generation of long pulse and high power $\mathrm{THz}$, required for $\mathrm{THz}$ communication, remote sensing and ultrafast material characterization.

Tightly confined guided electromagnetic waves are used as a probing radiation and having large number of applications [16-24], known as surface plasmons (SPs). SPs are guided electromagnetic modes propagate along the interface between a conductor and a dielectric or conductor and air with their field amplitude falling off exponentially away from it in either medium [25-27]. The structured surfaces, also support SPs at THz frequency range are called designer plasmonic structures or 'spoof' SPs structures [28-30]. Two structures, an ultrathin metal film dielectric plate, and a metal coated optical fiber, are shown to support low loss $\mathrm{THz}$ SPs $[31,32]$. These structures are shown to support $\mathrm{THz}$ SPs with weak attenuation. But the 
metal has loss and low conferment of SPs as compared to graphene plasmons (GPs) at $\mathrm{THz}$ frequencies. So graphene is a most suitable candidate for $\mathrm{THz}$ radiation generation.

Graphene is a 2D material, which has unique properties and potential applications for making the nano devices [33-40]. The most striking feature of graphene is that all its Dirac electrons, have same speed, as energy versus momentum relation is linear, irrespective of their energy. In graphene the localized electron motion in a plane with very small electron effective mass giving rise to high in-plane conductivity. The two dimensional nature of graphene also supports plasmons with wavelengths substantially smaller than free-space electromagnetic radiation of the same frequency by approximately two orders of magnitude and generating large non-local effects. Graphene plasmons (GPs) are used for making the compact radiation sources on chip in infrared-visible-ultraviolet range [41].

The difference frequency generation (DFG) at mid-infrared is enhanced due to the presence of plasmons resonance [42-45]. The graphene holds a great promise for designing the plasmonic sensors or new THz sources. An all-optical coupling scheme is used for plasmon generation in graphene. An electron beam is used for coherent and tunable $\mathrm{THz}$ radiation generation from multilayer graphene deposited on a substrate [46]. Despite the recent intensive research, generation of coherent and tunable $\mathrm{THz}$ radiation remains a significant challenge.

In this manuscript, we put forth the new and efficient scheme of THz GPs generation via the nonlinear mixing of two laser beams in the graphene coated optical fibre. The fiber has a dielectric ripple (volume grating) of wave number q. The ripple imparts spatial harmonics to lasers shifted by q. The lasers and their spatial harmonics exert a difference frequency ponderomotive force on film electrons with wave number $k_{z}=k_{1 z}-k_{2 z}+q$, where $k_{1 z}$ and $k_{2 z}$ are the 
wave numbers of the lasers. The ponderomotive force drives the THz GPs when q satisfies the phase matching condition. In section II, we developed the formalism of THz GPs in graphene coated fiber and the nonlinear mixing of lasers and THz excitation is discussed in section III. We discussed the results in section IV. 


\section{THz Graphene Plasmons in Graphene Coated Optical Fibre}

Consider an optical fiber (cf.1) of radius ' $a$ ' is coated by graphene sheet of conductivity $\sigma_{g}$. Consider the thin film of graphene is a layer zero thickness and infinite conductivity and the current density in graphene layer may be defined as

$$
J=\left.\sigma_{g} d \delta(r-a) E_{z}\right|_{r=a} \hat{z}=\left.\sigma_{g} d E_{z}\right|_{r=a} \hat{z}
$$

The graphene plasmons (GPs) propagate along $\hat{z}$ direction of fiber i.e. length of fiber with variation as $\exp \left[-i\left(\omega t-k_{z} z\right)\right]$ is shown in Fig.1. The electric and magnetic fields of the GPs in different regions can be written as for $r<a$ (Glass) region 1

$$
\begin{aligned}
& E_{1 z}=A_{01} I_{0}\left(\alpha_{1} r\right) \exp \left[-i\left(\omega_{1} t-k_{1 z} z\right)\right], \\
& H_{1 \varphi}=\frac{\omega \varepsilon_{0} \varepsilon_{1}}{i \alpha_{1}} A_{01} I_{0}^{\prime}\left(\alpha_{1} r\right) \exp \left[-i\left(\omega_{1} t-k_{1 z} z\right)\right],
\end{aligned}
$$

where $\alpha_{1}^{2}=k_{z}^{2}-\frac{\omega^{2}}{c^{2}} \varepsilon_{1}$.

For region $r>a$ (vacuum) region 2

$$
\begin{aligned}
& E_{2 z}=A_{02} K_{0}\left(\alpha_{2} r\right) \exp \left[-i\left(\omega_{2} t-k_{2 z} z\right)\right], \\
& H_{2 \varphi}=-\frac{\omega \varepsilon_{0} \varepsilon_{2}}{i \alpha_{2}} A_{02} K_{0}^{\prime}\left(\alpha_{2} r\right) \exp \left[-i\left(\omega_{2} t-k_{2 z} z\right)\right],
\end{aligned}
$$

where $\alpha_{2}^{2}=k_{z}^{2}-\frac{\omega^{2}}{c^{2}} \varepsilon_{2}$. Using jump condition on $\vec{H}($ at $r=a)$,

$$
\left.\vec{H}_{\phi}\right|_{2}-\left.\vec{H}_{\phi}\right|_{1}=\left.\sigma_{g} d E_{z}\right|_{r=a}
$$

Putting the Eqs. (2)\& (3) into Eq. (4), one may get

$$
\frac{\varepsilon_{2}}{\alpha_{2}} \frac{K_{0}^{\prime}\left(\alpha_{2} a\right)}{K_{0}\left(\alpha_{2} a\right)}-\frac{\varepsilon_{1}}{\alpha_{1}} \frac{I_{0}^{\prime}\left(\alpha_{1} a\right)}{I_{0}\left(\alpha_{1} a\right)}=-\frac{i \sigma_{g} d}{\omega \varepsilon_{0}}
$$

The Eq. (5) gives the GPs dispersion relation of SPs in graphene. In Fig. (2), we have plotted the normalized frequency $\omega / \omega_{p}$ versus normalized wave vector $k_{z} c / \omega_{p}$ for two 
normalized radii $a \omega_{p} / c=10^{-2}, a \omega_{p} / c=10^{-3}$ of optical fibre, normalized graphene sheet thickness $d \omega_{p} / c=0.08$ and for typical parameters:, $v_{2}=10^{6} \mathrm{~m} / \mathrm{s} ; c=3 \times 10^{8} \mathrm{~m} / \mathrm{s}$. The figure shows that with increasing the radius of optical fibre the coupling is between lasers and graphene plasmons becomes weak. As the frequency increases with wave number however; the phase velocity of graphene plasmons slows down with increasing the frequency.

\section{III.Nonlinear Mixing of Two Laser Beams and THz Surface Plasmons Generation in Graphene Coated Optical Fibre}

Let two laser beams are propagate in the azimuthally symmetric mode inside the graphene coated fiber (Fig.2). The electric fields of the laser beams inside the fiber are

$$
\begin{aligned}
& \vec{E}_{1}=A_{1}\left[\hat{z} \vec{J}_{0}\left(k_{1 \perp} r\right)+i \hat{r} \frac{k_{1 z}}{k_{1 \perp}} J_{0}^{\prime}\left(k_{1 \perp} r\right)\right] \exp \left[-i\left(\omega_{1} t-k_{1 z} z\right)\right] \\
& \vec{E}_{2}=A_{2}\left[\vec{z}_{0}\left(k_{2 \perp} r\right)+i \hat{r} \frac{k_{2 z}}{k_{2 \perp}} J_{0}^{\prime}\left(k_{2 \perp} r\right)\right] \exp \left[-i\left(\omega_{2} t-k_{2 z} z\right)\right]
\end{aligned}
$$

The fiber to have a dielectric constant ripple

$$
\varepsilon_{g}=\varepsilon_{g 0}+\varepsilon_{g q}, \varepsilon_{g q}=\varepsilon_{g 2} e^{i q z}
$$

The displacement vector inside the fiber is $\overrightarrow{\mathrm{D}}_{1}=\varepsilon_{\mathrm{g}} \overrightarrow{\mathrm{E}}_{1}=\varepsilon_{\mathrm{g} 0} \overrightarrow{\mathrm{E}}_{1}+\frac{1}{2} \varepsilon_{\mathrm{gq}} \overrightarrow{\mathrm{E}}$ and acquires a component that goes as $\mathrm{e}^{-\mathrm{i}\left[\omega_{1} \mathrm{t}-\left(\mathrm{k}_{12}+\mathrm{q}\right) z\right]}$. This component produces an electromagnetic field (in compliance with $\nabla \cdot \vec{D}=0)$

$\vec{E}_{1+} \cong-\frac{\varepsilon_{g q}}{2 \varepsilon_{g 0}} \vec{E}_{1} \approx \exp \left[-i\left\{\omega_{1} t-\left(k_{1 z}+q_{z}\right) z\right\}\right]$

Similarly,

$\vec{E}_{2+} \cong-\frac{\varepsilon_{g q}}{2 \varepsilon_{g 0}} \vec{E}_{2} \approx \exp \left[-i\left\{\omega_{2} t-\left(k_{2 z}+q_{z}\right) z\right\}\right]$

Here, the phase matching conditions demands that 
$\omega_{S P T H z}=\omega_{1}-\omega_{2}, k_{S P T H z}=k_{1 z}-k_{2 z}+q$

The lasers impart oscillatory velocities to the electrons, given as

$\vec{v}_{1+}=\frac{e \vec{E}_{1+}}{m i \omega_{1}}, \quad \vec{v}_{2+}=\frac{e \vec{E}_{2+}}{m i \omega_{2}}$

These laser beams also impart the ponderomotive force $F_{p z}$ on electrons at beat frequency given by

$F_{p z} \approx \frac{m i\left(k_{1 z}-k_{2 z}+q\right)}{2} \vec{v}_{1} \vec{v}_{2}^{*} \frac{\varepsilon_{g q}}{2 \varepsilon_{g 0}}$

This ponderomotive force also imparts oscillatory velocity to electrons at frequency $\left(\omega_{1}-\omega_{2}\right)$ and may be written as

$\vec{v}_{\omega}=\frac{\hat{z} F_{p z}}{-i m \omega}$,

the nonlinear current in graphene sheet may be written as

$\vec{J}_{\omega}^{N L}=-\hat{z} \frac{n_{0} e\left(k_{1 z}-k_{2 z}+q\right)}{2 \omega} \vec{v}_{1} \vec{v}_{2}^{*} \frac{\varepsilon_{g q}}{2 \varepsilon_{g 0}} \cong \hat{z} F d \delta(r) \exp \left[-i\left(\omega_{S P} t-k_{S P} z\right)\right]$

where $\vec{F}=\frac{n_{0} e k_{s p}}{4 \omega} \frac{e^{2}\left|E_{1}\right|\left|E_{2}\right|}{m^{2} \omega_{1} \omega_{2}} \frac{\varepsilon_{g q}}{\varepsilon_{g 0}}$.

This nonlinear current density is localized in graphene sheet, which is the source of $\mathrm{THz}$ graphene plasmons. The electric and magnetic field vectors for GPs are given as

For $r<a$ (glass)

$\vec{E}_{1}=A_{1}\left[\hat{z} \vec{I}_{0}\left(\alpha_{1} r\right)-i \hat{r} \frac{k_{z}}{\alpha_{1}} I_{0}^{\prime}\left(\alpha_{1} r\right)\right] \exp \left[-i\left(\omega_{1} t-k_{1} z\right)\right]$

$\vec{H}_{\phi}=\frac{i \omega \varepsilon_{0} \varepsilon_{1}}{\alpha_{1}} A_{1} I_{0}^{\prime}\left(\alpha_{1} r\right) \exp \left[-i\left(\omega_{1} t-k_{1} z\right)\right]$

For $r>a($ vacuum) 


$$
\begin{aligned}
& \vec{E}_{2}=A_{2}\left[\hat{z} \vec{K}_{0}\left(\alpha_{2} r\right)-i r \frac{k_{z}}{\alpha_{2}} K_{0}^{\prime}\left(\alpha_{2} r\right)\right] \exp \left[-i\left(\omega_{2} t-k_{2} z\right)\right], \\
& \vec{H}_{\varphi}=-\frac{i \omega \varepsilon_{0}}{\alpha_{2}} A_{2} K_{0}^{\prime}\left(\alpha_{2} r\right) \exp \left[-i\left(\omega_{2} t-k_{2} z\right)\right] .
\end{aligned}
$$

The nonlinear current density manifests itself in the jump condition on the $\vec{H}_{\phi}$ component of the GPs at $r=a$, gives

$$
\begin{aligned}
& D A_{0}=\frac{-i F d / \omega \varepsilon_{0}}{K_{0}^{\prime}\left(\alpha_{2} a\right)}, \\
& D=\frac{\varepsilon_{1}}{\alpha_{1}} \frac{I_{0}^{\prime}\left(\alpha_{1} a\right)}{I_{0}\left(\alpha_{1} a\right)}-\frac{1}{\alpha_{2}} \frac{K_{0}^{\prime}\left(\alpha_{2} a\right)}{K_{0}\left(\alpha_{2} a\right)}-\frac{\sigma_{g} d}{i \omega \varepsilon_{0}} .
\end{aligned}
$$

Solving Eq. (19), it yields the amplitude of THz GPs

$$
A_{0}=\frac{F d L / \omega \varepsilon_{0}}{K_{0}^{\prime}\left(\alpha_{2} a\right) \partial D / \partial k_{s p}}
$$

At exact phase matching condition, D is vanishes. However due to finite length of interaction $L=2 r_{0} \quad$ where $r_{\mathbf{O}}$ is laser spot size, so $\mathrm{D}$ can be replaced by region $D \cong-i\left(\partial D / \partial k_{z}\right)(\partial / \partial z)$, Eq. (19) can be written as

$$
\frac{\partial D}{\partial k_{z}}=-k_{z}\left[\frac{\varepsilon_{1}}{\alpha_{1}^{3}} \frac{I_{0}^{\prime}\left(\alpha_{1} a\right)}{I_{0}\left(\alpha_{1} a\right)}-\frac{1}{\alpha_{2}^{3}} \frac{K_{0}^{\prime}\left(\alpha_{2} a\right)}{K_{0}\left(\alpha_{2} a\right)}\right] .
$$

Solving and rearranging the terms in Eq. (22), we have

$$
\left|\frac{A_{0}}{A_{1}}\right|=\frac{1 /\left(\omega / \omega_{p}\right)\left(\omega / 4 \omega_{1}\right)\left(k_{S P} / k_{z}\right)\left(\left|v_{2}\right| / c\right)\left(\varepsilon_{g q} / \varepsilon_{g 0}\right)\left(d \omega_{p} / c\right)(L \omega / c)}{K_{0}^{\prime}\left(\alpha_{2} a\right)\left(\frac{\varepsilon_{1}}{\left(k_{z}^{2}-\left(\omega^{2} / c\right) \varepsilon_{1}\right)^{3 / 2}} \frac{I_{0}^{\prime}\left(\alpha_{1} a\right)}{I_{0}\left(\alpha_{1} a\right)}-\frac{1}{\left(\omega^{2} / c\right)^{3 / 2}} \frac{K_{0}^{\prime}\left(\alpha_{2} a\right)}{K_{0}\left(\alpha_{2} a\right)}\right)} .
$$

Eq. (23) gives the amplitude of terahertz graphene plasmon. For following parameters: $v_{2}=10^{6} \mathrm{~m} / \mathrm{s} ; \quad c=3 \times 10^{8} \mathrm{~m} / \mathrm{s} ; \quad \omega=10^{13} \mathrm{rad} / \mathrm{s} ; k_{z}^{2} c^{2} / \omega^{2}=2.6 ; \omega_{1}=2 \times 10^{12} \mathrm{rad} / \mathrm{s} ;$ $\omega_{p} \approx 2.5 \times 10^{12} \mathrm{rad} / \mathrm{s}$, normalized thickness $d \omega_{p} / c=0.08,0.10,0.12$, normalized radius of optical 
fibre $a \omega_{p} / c=10^{-2}, 10^{-3} L \omega / c=10^{3}, \varepsilon_{g^{2}} / \varepsilon_{g 0}=0.2$.For $\mathrm{CO}_{2}$ laser, $e\left|E_{2}\right| / m \omega_{2} c=10^{-2}$, corresponds to an intensity $3 \times 10^{12} \mathrm{~W} / \mathrm{cm}^{2}$ and power $\sim \mathrm{MW}$. For $1 \mu \mathrm{m}$, wavelength it corresponds to an intensity of $3 \times 10^{14} \mathrm{~W} / \mathrm{cm}^{2}$. Fig. 4, 5 and 6 , show that as the frequency raises, the normalized amplitude of THz SPW decreases. It is due to the weakening of the nonlinear coupling of SPs at higher frequencies.

\section{Conclusion}

In conclusion, the graphene coated fiber offers the promise of producing phase matched THz graphene plasmons using laser moderate power (MW range) and intensity. The nonlinearity arises through the ponderomotive force and phase matching is provided by the volume grating of suitable wave number inside the fiber. The efficiency is one order of magnitude smaller than the ideal efficiency given by Manley Rowe relations. The thickness of the graphene sheet and radius of an optical fibre are important parameters to influence the $\mathrm{THz}$ graphene surface plasmons resonant frequency. The normalized amplitude of THz GPS decrease with frequency as the nonlinear coupling gets weaker. The fiber configuration is more suitable as the grating structures in optical fibers are routinely available. The efficiency of the device is around $0.01 \%$ at a laser intensity of $3 \times 10^{14} \mathrm{~W} / \mathrm{cm}^{2}$.

Funding: Not applicable

Conflicts of interest/Competing interests: Not applicable

Data available in article: The data that supports the findings of this study are available within the article.

Code availability: Not applicable

Authors Contributions: All authors contributed equally to this work. 
Ethics approval: Not applicable

Consent to participate: Not applicable

Consent for publication: Not applicable 


\section{References}

1. B. Ferguson and X.-C. Zhang, Nat Mater. 1, 26(2002).

2. R. Huber, F. Tauser, A. Brodschelm, M. Bichler, G. Abstreiter, and A. Leitenstorfer, Nature. 414, 286( 2001).

3. M. Nagel, M. Brucherseifer, P. H. Bolivar, H. Kurz, A. Bosserhoff, and R. Buttner, Appl. Phys. Lett.Appl. Phys. Lett. 80, 154(2002).

4. D. Grischkowsky, Søren Keiding, Martin van Exter, and Ch. Fattinger, J. Opt. Soc. Am. B 7, 2006(1990).

5. D. M. Mittleman, R.H.Jacobsen, M.C.Nuss, IEEE J. Selected Topics in Quantum Electronics 2, 679(1996).

6. M. C. Beard, G. M. Turner, and C. A. Schmuttenmaer, J. Appl. Phys. 90, 5915 (2001).

7. J. Federici and L. Moeller, Y. C. Shen, T. Lo, P. F. Taday, B. E. Cole, W. R. Tribe, and M. C. Kemp, Appl. Phys. Lett. 86, 241116(2005).

8. P. Spangle, J. R. Penano, B. Hafizi and C. A. Kapetanakas, Phy. Rev. E 69, 066415 (2004).

9. J. Zhao, Y. Zhang, Z. Wang, W. Chu, B. Zeng, W. Liu, Y. Z. Cheng and Xu, Laser Phys. Lett. 11, 095302 (2014).

10. P. Cicènas, A. Geižutis, V. L. Malevich, and A. Krotkus, Opt. Letts. Vol. 40, Issue 22, $5164(2015)$.

11. X.-C. Zhang, Y. Jin, L. E. Kingsley, and M. Weiner, Appl. Phys. Lett. 62, 2477 (1993).

12. H. Hamster, A. Sullivan, S. Gordon, W. White, and R. W. Falcone, Phys. Rev. Lett. 71, 2725(1993).

13. T.M. Antonsen, Jr., J. Palastro Howard M. Milchberg, Phys. Plasmas 14, 033107(2007) 
14. Xu Xie, Jingzhou Xu, Jianming Dai, and X.-C. Zhang, Appl. Phys. Lett. 90, 141104(2007).

15. D. J. Cook and R. M. Hochstrasser, Opt. Lett. 25, 1210(2000).

16. E. Ozbay, Science 311, 189 (2006).

17. Barnes, A. Dereux and T. W. Ebbesen, Nature 424, 824 (2003).

18. Liu, H. Lee, Y. Xiong, C. Sun and X. Zhang, Science 315, 1686(2007).

19. S. Kim, J. Jin, Y.-J. Kim, I.-Y. Park, Y. Kim and S.W. Kim, Nature 453,757 (2008).

20. I. Bozhevolnyi, V. S. Volkov, E. Devaux, J.-Y. Laluet and T. W. Ebbesen, Nature 440, 508 (2006).

21. P. Kumar, V. K. Tripathi and C. S. Liu, J. Appl. Phys. 104, 033306 (2008).

22. D. J. Bergman and M. I. Stockman, Phys. Rev. Lett. 90, 027402 (2003).

23. K. R. Catchpole and A. Polman, Opt. Express 16, 21793 (2008).

24. Homola, S. S. Yee and G. Gauglitz, Sens. Actuators, B 54, 3 (1999).

25. H. Raether, Surface Plasmons on Smooth and Rough Surfaces and on Gratings (Springer, Berlin, 1988).

26. D. Boardman, Electromagnetic Surface Modes (Wiley, New York, 1982).

27. S. A. Maier, Plasmonics: Fundamentals and Applications (Springer-Verlag, New York, 2007).

28. J. B. Pendry, L. Martin-Moreno, and F. J. Garcia-Vidal, Science 305, 847(2004).

29. F. J. Garcia-Vidal, L. Martin-Moreno, and J. B. Pendry, J. Opt. A: Pure Appl. Opt.7, S97 (2005).

30. C. R. Williams, S. R. Andrews, S. A. Maier, A. I. Fernandez-Domınguez, L. MartınMoreno and F. J. Garcia-Vidal, Nature Photon. 2, 175 (2008). 
31. Pawan Kumar and V. K. Tripathi, J. Appl. Phys. 114, 053101 (2013).

32. Pawan Kumar and V. K. Tripathi, Opt. Lett. 38, Issue 18, 3475(2013)

33. K. S. Novoselov, A. K. Geim, S.V. Morozov, D. Jiang, M. I. Katsnelson, I.V. Grigorieva, S.V. Dubonos, and A. A. Firsov, Nature 438, 197 (2005).

34. Y. Zhang, Y.-W. Tan, H. L. Stormer and P. Kim, Nature 438, 201 (2005).

35. A. K. Geim, Science 324, 1530 (2009).

36. D.N. Basov, M.M. Fogler, A. Lanzara, F. Wang and Y. Zhang, Rev. Modern Phys. 86, 959 (2014).

37. A.K. Geim and K.S. Novoselov, Nat. Mater. 6, 183 (2007).

38. P. R. Wallace, Phys. Rev. 71, 622 (1947).

39. A. H. Castro Neto, F. Guinea, N. M. R. Peres, K. S. Novoselov, and A. K. Geim, Rev. Mod. Phys. 81, 109 (2009)

40. A.N. Grigorenko, M. Palini and K.S. Novoselov, Nat. Photonics 6, 749 (2012).

41. L. J. Wong, I. Kaminer, O. Ilic, J. D. Joannopoulos, and M. Soljačić, Nat. Photonics 10, 46 (2016).

42. T. J. Constant, S. M. Hornett, D. E. Chang, and E. Hendry, Nat. Phys. 12, 124 (2015).

43. B. Yao, Y. Liu, S.-W. Huang, C. Choi, Z. Xie, J. F. Flores, Y. Wu, M. Yu, D.-L. Kwong, Y. Huang, Y. Rao, X. Duan, and C. W. Wong, Nat. Photon. 12, 22 (2018).

44. X. H. Yao, M. Tokman, and A. Belyanin, Phys. Rev. Lett. 112, 055501 (2014).

45. Neha Verma, Anil Govindan and Pawan Kumar, Plasmonics 15, Issue 6, November 2020.

46. S. Liu, C. Zhang, M. Hu, X. Chen, P. Zhang, S. Gong, T. Zhao, and R. Zhong, Appl. Phys. Lett. 104, 201104 (2014). 


\section{Figure Captions}

Fig.1 THz GPs propagation in graphene coated optical fiber.

Fig.2. Dispersion relation for graphene plasmons.

Fig.3. THz GPs excitation via nonlinear mixing of lasers in graphene coated optical fiber.

Fig.4 .Normalized $\mathrm{THz}$ graphene surface plasmons amplitude variation with normalized frequency for different two different normalized radii of optical fibre and fixed thickness of graphene sheet.

Fig.5. Normalized $\mathrm{THz}$ graphene surface plasmons amplitude variation with normalized frequency for different normalized thickness of graphene sheet and fixed normalized radius of optical fibre.

Fig.6. Normalized $\mathrm{THz}$ graphene surface plasmons amplitude variation with normalized frequency for different normalized thickness of graphene sheet and fixed normalized radius of optical fibre. 


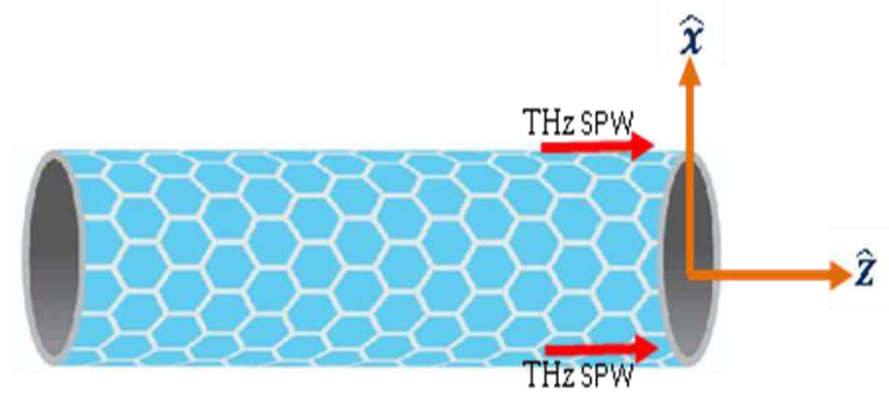

Fig.1 THz GPs propagation in graphene coated optical fiber.

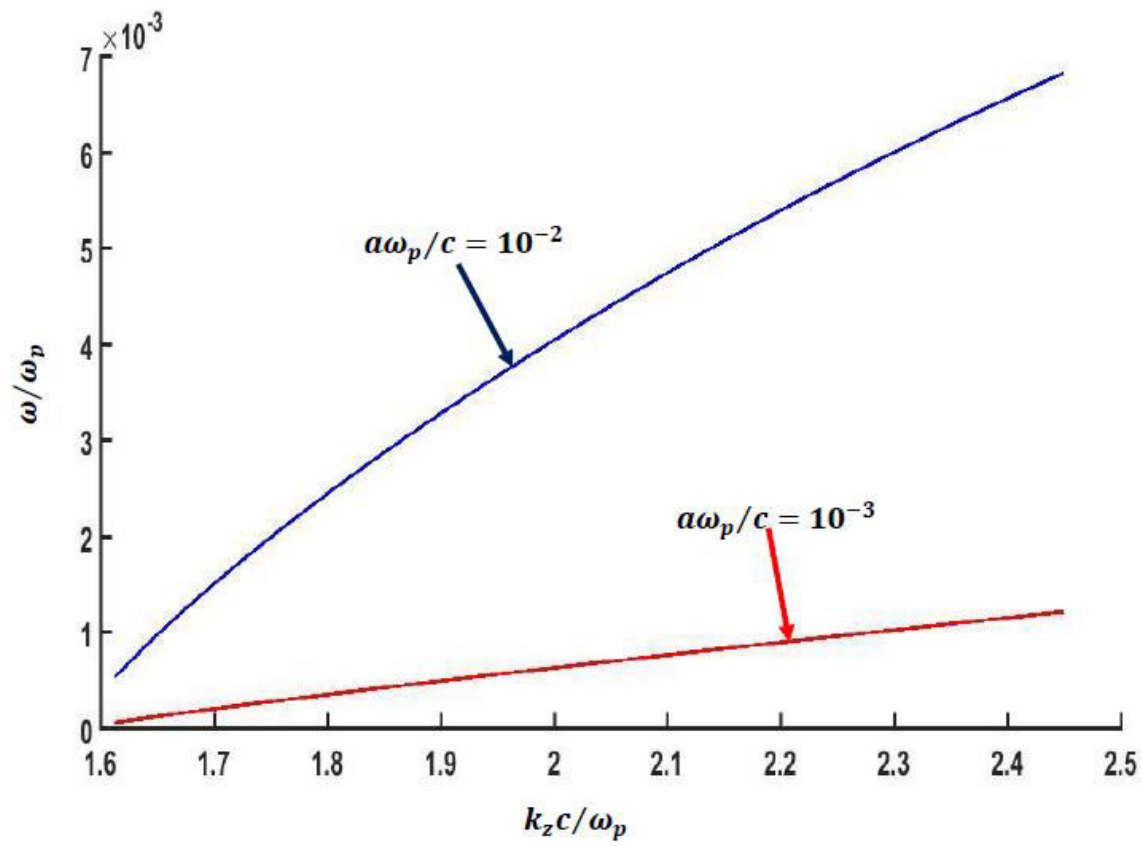

Fig.2. Dispersion relation for graphene plasmons. 


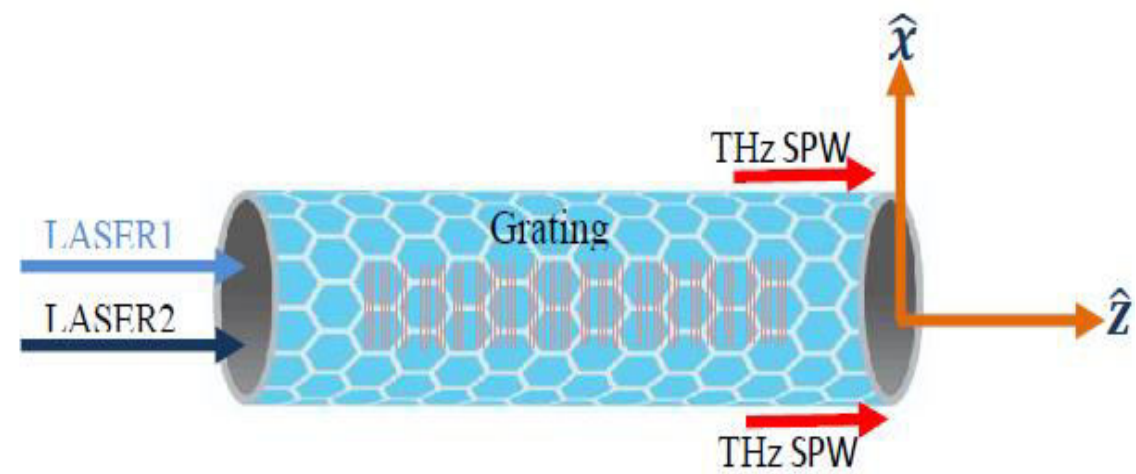

Fig.3. THz GPs excitation via nonlinear mixing of lasers in graphene coated optical fiber.

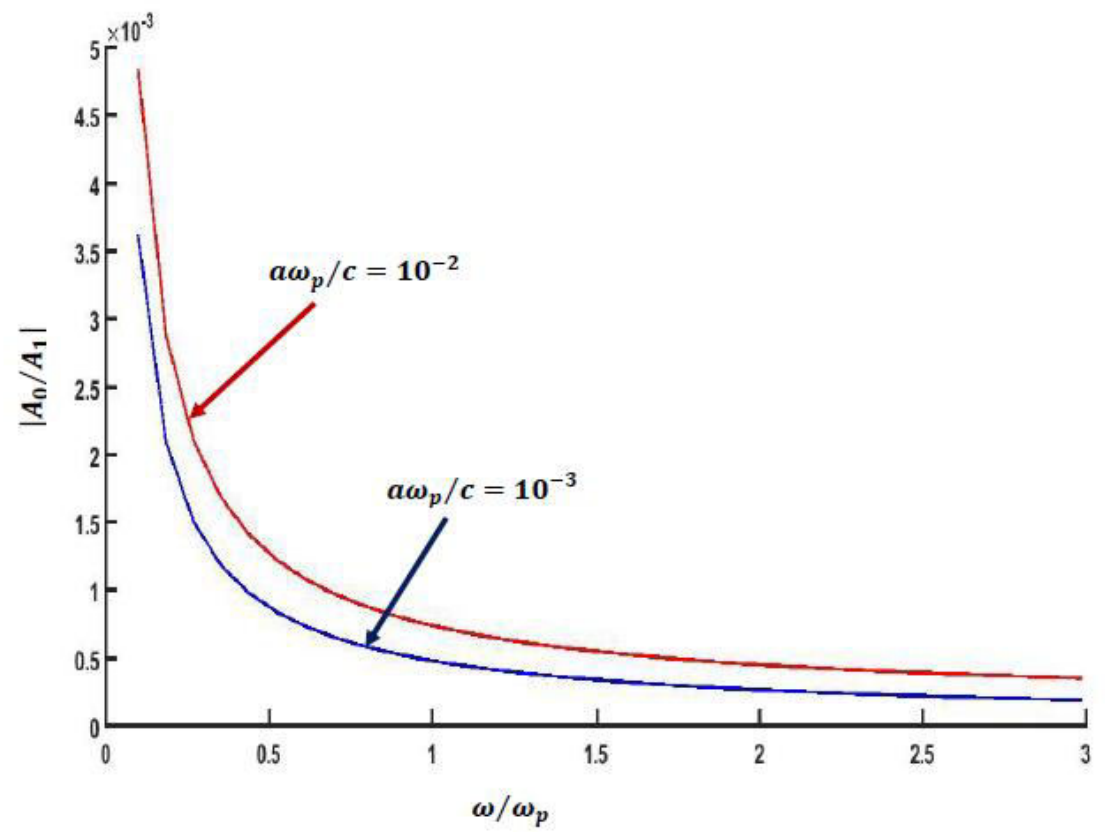

Fig.4. Normalized $\mathrm{THz}$ graphene surface plasmons amplitude variation with normalized frequency for different two different normalized radii $a \omega_{p} / c=10^{-2}, 10^{-3}$ of optical fibre and fixed thickness of graphene sheet. 


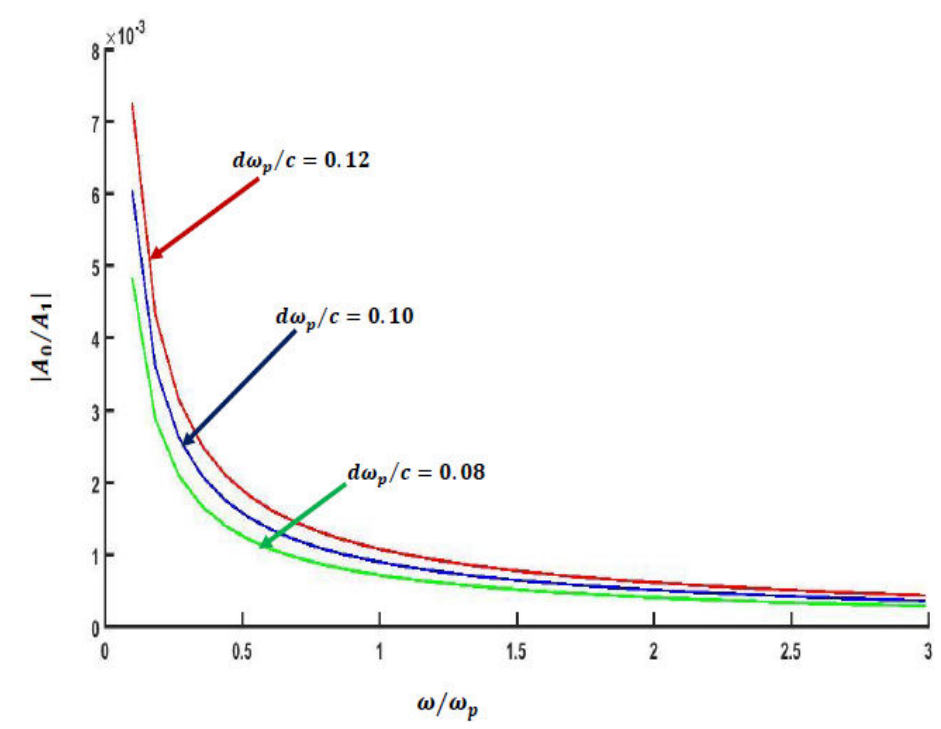

Fig.5. Normalized $\mathrm{THz}$ graphene surface plasmons amplitude variation with normalized frequency for different normalized thickness of graphene sheet and fixed normalized radius $a \omega_{p} / c=10^{-2}$ of optical fibre.

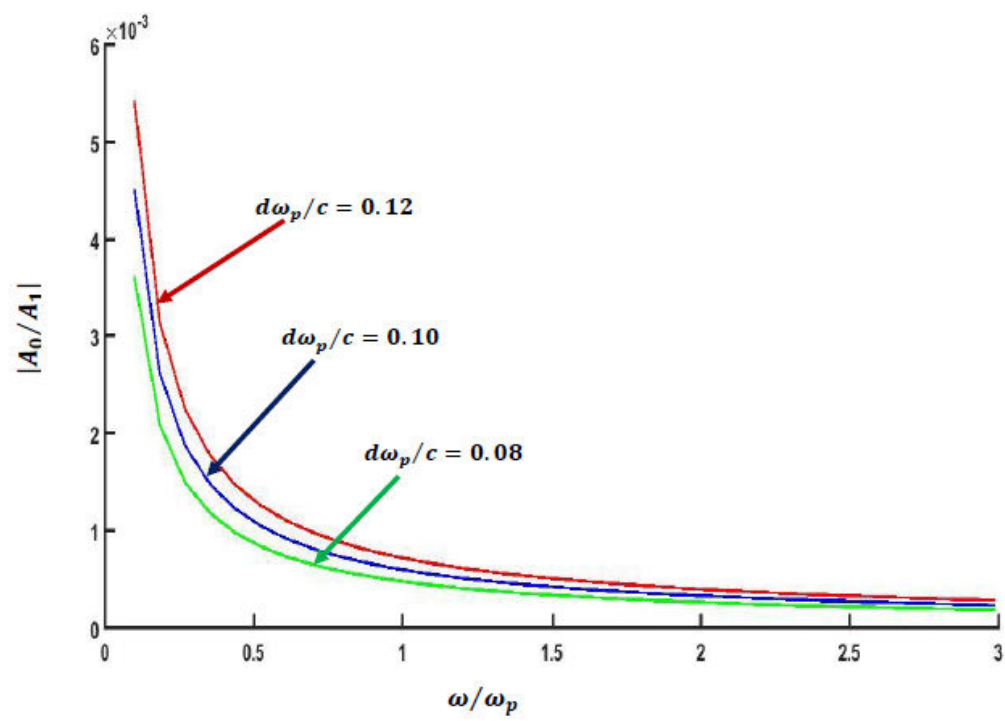

Fig.6. Normalized $\mathrm{THz}$ graphene surface plasmons amplitude variation with normalized frequency for different normalized thickness of graphene sheet and fixed normalized radius $a \omega_{p} / c=10^{-3}$ of optical fibre. 